\title{
BMJ Open Canada-wide mixed methods analysis evaluating the reasons for inappropriate emergency department presentation in patients with a history of atrial fibrillation: the multicentre AF-ED trial
}

To cite: Hong KL, Babiolakis C, Zile B, et al. Canada-wide mixed methods analysis evaluating the reasons for inappropriate emergency department presentation in patients with a history of atrial fibrillation: the multicentre AF-ED trial. BMJ Open 2020;10:e033482. doi:10.1136/ bmjopen-2019-033482

- Prepublication history for this paper is available online. To view these files, please visit the journal online (http://dx.doi. org/10.1136/bmjopen-2019033482).

Received 27 September 2019 Revised 14 February 2020 Accepted 12 March 2020

Check for updates

(C) Author(s) (or their employer(s)) 2020. Re-use permitted under CC BY-NC. No commercial re-use. See rights and permissions. Published by BMJ.

For numbered affiliations see end of article.

Correspondence to

Dr Benedict M Glover;

benedict.glover@sunnybrook.ca

\section{ABSTRACT}

Objectives The primary objective of this study was to ascertain the reasons for emergency department (ED) attendance among patients with a history of atrial fibrillation (AF).

Design Appropriate ED attendance was defined by the requirement for an electrical or chemical cardioversion and/or an attendance resulting in hospitalisation or administration of intravenous medications for ventricular rate control. Quantitative and qualitative responses were recorded and analysed using descriptive statistics and content analysis, respectively. Random effects logistic regression was performed to estimate the OR of inappropriate ED attendance based on clinically relevant patient characteristics.

Participants Participants $\geq 18$ years with a documented history of AF were approached in one of eight centres partaking in the study across Canada (Ontario, Nova Scotia, Alberta and British Columbia).

Results of the 356 patients enrolled $(67 \pm 13,45 \%$ female), the majority $(271 / 356,76 \%)$ had inappropriate reasons for presentation and did not require urgent $E D$ treatment. Approximately $50 \%$ of patients( $172 / 356$, $48 \%$ ) were driven to the ED due to symptoms, while the remainder presented on the basis of general fear or anxiety $(67 / 356,19 \%)$ or prior medical advice (117/356, $33 \%)$. Random effects logistic regression analysis showed that patients with a history of congestive heart failure were significantly more likely to seek urgent care for appropriate reasons $(p=0.03)$. Likewise, symptomrelated concerns for ED presentation were significantly less likely to result in inappropriate visitation $(p=0.02)$. When patients were surveyed on alternatives to ED care, the highest proportion of responses among both groups was in favour of specialised rapid assessment outpatient clinics (186/356, 52\%). Qualitative content analysis confirmed these results.

Conclusions Improved education focused on symptom management and alleviating disease-related anxiety as well as the institution of rapid access arrhythmias
Strengths and limitations of this study

To our knowledge, this is the largest prospective, multicentre, mixed methods analysis evaluating patient-specific reasons for inappropriate attendance to the emergency department.

- This study combines both quantitative and qualitative data that permit a more complete and synergistic application of data.

- All patients were interviewed using a standardised approach in order to minimise the impact of interobserver variability.

- The use of a survey-based approach to data collection is susceptible to reporting bias.

- This multicentre study was based on voluntary participation from all centres, and all patients who agreed to take part may not represent the entire patient population.

clinics may reduce the need for unnecessary healthcare utilisation in the ED and subsequent hospitalisation.

Trial registration number NCT03127085

\section{INTRODUCTION}

Atrial fibrillation (AF) is the most common sustained cardiac arrhythmia encountered in clinical practice and is associated with increasing morbidity and mortality. ${ }^{1-3}$ Given its increasing prevalence as an age-dependent disease $^{45}$ as well as associated cardiovascular complications such as stroke and heart failure, the impact of elevated healthcare utilisation incurred from AF-related emergency department (ED) presentation poses a global healthcare burden. ${ }^{6}$ This is particularly relevant when higher healthcare utilisation is required for inappropriate ED presentation, 
given that only approximately $15 \%$ of Canadian patients with primary AF are admitted to hospital. ${ }^{7}$

In the Canadian population, the overall rate of AF-related hospitalisations is estimated to be 583 per 100000 individuals, ${ }^{8}$ equating to more than $\$ 815$ million in hospital costs attributable to $\mathrm{AF}^{9}$ However, rates of hospital admission have been found to be as high as $40 \%$ in Ontario, with notable variability between centres. ${ }^{10}$ Given this, guidelines suggest that hospitalisation should be reserved for patients who are either highly symptomatic or haemodynamically unstable ${ }^{1112}$ and that the remainder may receive further medical treatment outside the ED environment.

Despite the fact that a sizeable percentage of patients with an established diagnosis of AF attend the ED with symptoms perceived to be related to their underlying arrhythmia, many of these attendances may not necessarily warrant emergent care. These inappropriate ED visits may be safely reduced through alternative management strategies, resulting in significant reductions in healthcare utilisation and subsequent hospitalisation.

We, therefore, used a patient-oriented approach to assess the reasons for ED attendance in the setting of $\mathrm{AF}$ and to determine factors for inappropriate visitation. These results were then used to evaluate patient preferences with regards to treatment strategies that may be implemented as safe and effective alternatives to ED intervention.

\section{METHODS}

\section{Participant survey}

All patients with an established diagnosis of AF who presented to any of the participating eight academic or community EDs across Canada were approached for enrolment into this multicentre study. All index ED visits were patient initiated as a result of symptoms or concerns perceived to be related to their diagnosis of AF. Attendances occur either as a result of self-reporting or following a call to the emergency services. All patients who attend the ED in Canada are reviewed and triaged by a nurse and a medical doctor, and further management is based on their plan. Patients were not invited to take part if they had other significant comorbidities that resulted in their ED attendance.

Patients were provided with a written invitation and consent form, and only those who completed this following their admission to the ED and mailed it to the recruitment centre were called. All participants received a telephone interview from designated and suitably trained research personnel within 4 weeks of their index visit. Eligibility criteria included: (1) age $\geq 18$ years, (2) AF being the primary reason for the ED visit, (3) patients with an established history of AF (prior diagnosis documented on ECG or Holter strip) and (4) the ability to provide fully informed consent prior to the telephone interview. Patients were excluded if they were: (1) unable or unwilling to consent and participate, (2) enrolled in a concurrent cardiovascular drug or device research study and/or (3) had new-onset AF and/or (4) AF secondary to another condition that required active treatment.

Baseline demographic data including age, gender, AF type, existing comorbidities and medications were obtained from patient medical records. Information regarding their condition (symptoms associated with $\mathrm{AF}$, frequency of episodes, severity of symptoms, occurrence of palpitations, shortness of breath, exercise intolerance, lightheadedness and chest pain) and functional activity were obtained through a preformulated survey via telephone. Participants also provided explanations with regards to their reasons for ED attendance, acute interventions received in the ED and final disposition (hospital admission or ED discharge).

\section{Qualitative discussions}

Qualitative responses were recorded in order to further ascertain factors associated within appropriate ED visitation and alternative strategies that could be implemented to assist in the management of their AF. Individual responses to open-ended questions were analysed using a qualitative content analysis approach. Each statement was read in its entirety and coded word for word. Similar coded statements were then grouped together into categories and finally themes.

\section{Patient and public involvement}

The research questions and survey design was developed in collaboration with patient advisers as part of the Cardiac Arrhythmia Network of Canada. Engaging knowledge users at critical stages of the study ensured that key outcome measures would be relevant in guiding future clinical practice for $\mathrm{AF}$ and in the management of other chronic conditions.

\section{Statistical analysis}

Quantitative and qualitative responses were recorded and analysed using descriptive statistics and content analysis, respectively. With respect to quantitative analyses, continuous data were analysed using SAS V.9.4. Continuous variables were expressed as mean $\pm \mathrm{SD}$ and analysed using Student's t-test or non-parametrical tests (Wilcoxon and Mann-Whitney), as appropriate. Categorical variables were compared using the $\chi^{2}$ test, or Fisher's exact test, as appropriate. A two-tailed $\mathrm{p}$ value was used in all analyses, and a $p$ value $<0.05$ was considered statistically significant. Due to incomplete answers, missing variables and multiple answering options, observed numbers and percentages did not always add up to exactly $100 \%$. Random effects logistic regression was also performed to identify clinical characteristics associated with inappropriate ED attendance (outcome), with participating centres selected as the random intercepts. Logistic regression analysis was performed using SAS V.9.4, with the proc glimmix procedure for modelling. 


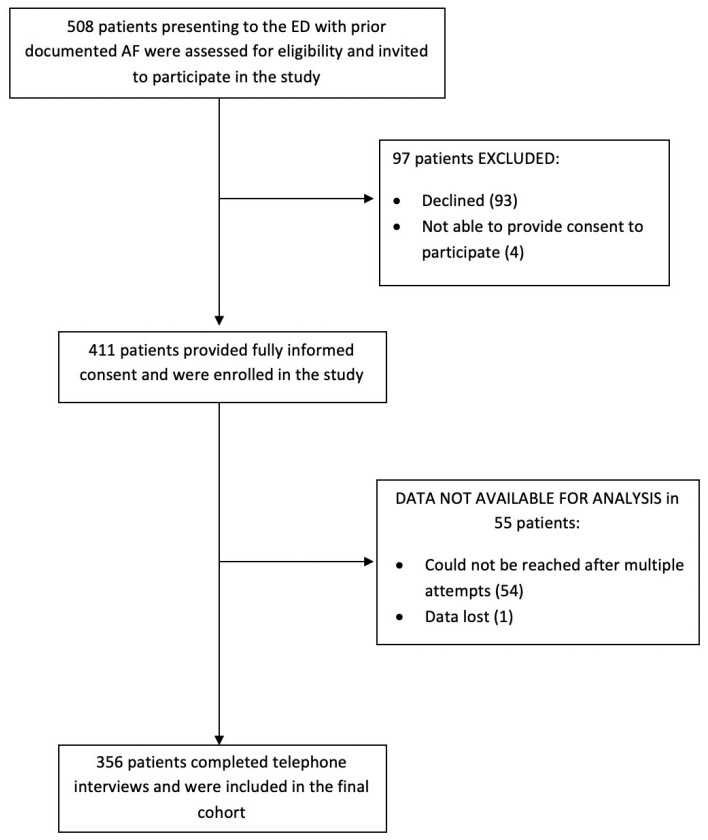

Figure 1 CONSORT diagram showing participant flow and final cohort. AF, atrial fibrillation; CONSORT, Consolidated Standards of Reporting Trials; ED, emergency department.

\section{Definitions}

AF type was defined as paroxysmal or persistent according to the recommendations of the 2016 European Society of Cardiology (ESC) Guidelines. ${ }^{13}$ Appropriate ED attendance was defined by the requirement for an electrical or chemical cardioversion and/or an attendance resulting in hospitalisation. Cardioversions were deemed appropriate if the patient had AF with haemodynamic instability in the form of angina pectoris, myocardial infarction and shock or pulmonary oedema. Electrical or chemical cardioversions performed for alternate reasons, as per the discretion of the physician, were not considered appropriate reasons for attendance.

\section{RESULTS \\ Survey}

Between August 2016 and August 2018, a total of 508 participants were approached to take part in this study. Of these, 97 patients were excluded (declined to participate/unable to provide fully informed consent), and data were unavailable for analysis in 55 patients (figure 1). The final cohort consisted of 356 patients (mean age $67 \pm 13$, $45 \%$ female) were enrolled across eight centres in four provinces (Ontario, Nova Scotia, Alberta and British Columbia) of which 172/356 (48\%) had symptomatic episodes. The majority of patients who were recruited to the study had attended an ED in an academic institution versus an ED based in a community hospital $(81 \%$ academic vs $19 \%$ community).
Baseline patient characteristics

Baseline characteristics, classification of $\mathrm{AF}$, medication use and existing cardiovascular comorbidities at ED presentation are displayed in table 1. Within this cohort, patients were stratified by appropriateness of their ED visit, of which 85/356 (24\%) had indications warranting emergency treatment, while the majority of patients $(271 / 356,76 \%)$ presented for inappropriate reasons thst may have been more effectively managed outside the ED setting. Within the appropriate group, 41/356 (12\%) underwent chemical cardioversion only, 16/356 (5\%) underwent an electrical cardioversion following an unsuccessful attempt at chemical cardioversion, 12/356 (3\%) and 16/356 (4\%) resulted in an admission into hospital for further management.

After performing logistic regression, the only baseline predictors of an appropriate ED attendance were as follows:

A history of diagnosed congestive heart failure (CHF) ( $18 \%$ vs $7 \%, \mathrm{p}=0.007)$.

A history of vascular disease (myocardial infarction with peripheral arterial disease) ( $16 \%$ vs $9 \%, \mathrm{p}=0.04)$.

Treatment with warfarin (20\% vs $10 \%, \mathrm{p}=0.03)$.

The type of AF, AF duration (from time of diagnosis), age and gender did not influence the appropriateness of ED visitation.

The proportion of patients receiving medical therapy at the time of their ED attendance is also displayed in table 1. On ED presentation, more than half of patients were receiving treatment with an oral anticoagulant (OAC) $(211 / 356,59 \%)$. Of these, a significantly higher proportion of patients with appropriate ED visits $(17 / 85,20 \%)$ were receiving warfarin at presentation as compared with those with inappropriate visits $(28 / 271,10 \%)(\mathrm{p}=0.03)$.

\section{Reasons for ED visitation and outcomes}

Approximately $50 \%$ of patients $(172 / 356,48 \%)$ were driven to the ED on the basis of symptoms. All other patients presented for reasons unrelated to their symptoms, namely on the basis of general fear or anxiety $(67 / 356,19 \%)$ or prior medical advice $(117 / 356,33 \%)$.

When presenting concerns were compared on the basis of visit appropriateness, the majority of patients receiving appropriate ED treatment did so on the basis of their AF-related symptoms $(71 / 85,84 \%)$ while only $101 / 271$ $(37 \%)$ of those in the inappropriate group presented on the basis of symptoms. As shown in figure 2, AF symptom reporting was found to be significantly correlated with appropriate ED attendance $(\mathrm{p}<0.0001)$. The most commonly reported symptom prompting patients to attend the ED, irrespective of visit appropriateness, were palpitations $(58 / 71,82 \%)$ followed by shortness of breath $(22 / 71,31 \%)$, chest tightness $(18 / 71,25 \%)$ and dizziness or lightheadedness $(14 / 71,20 \%)$. A note should be made that patients could report more than one symptom explaining why the percentage is greater than $100 \%$. Each of these symptoms was statistically more significant 
Table 1 Baseline demographics of the patient cohort presenting to the ED for AF-related reasons, stratified by appropriateness of visit

\begin{tabular}{|c|c|c|c|c|}
\hline & All patients & $\begin{array}{l}\text { Assessed as } \\
\text { appropriate }\end{array}$ & $\begin{array}{l}\text { Assessed as } \\
\text { inappropriate }\end{array}$ & \\
\hline & $\mathrm{n}=356$ & $n=85$ & $n=271$ & $P$ value \\
\hline Age $($ mean $\pm S D)$ & $67 \pm 13$ & $67 \pm 14$ & $67 \pm 13$ & NS \\
\hline Female (n (\%)) & $160(45)$ & $40(47)$ & $120(44)$ & NS \\
\hline $\begin{array}{l}\text { Duration of } \mathrm{AF} \text { from time of } \\
\text { initial diagnosis in months } \\
(\text { mean } \pm \mathrm{SD})\end{array}$ & $14( \pm 9)$ & $15( \pm 10)$ & $14( \pm 9)$ & NS \\
\hline \multicolumn{5}{|l|}{ Type of AF (n (\%)) } \\
\hline Paroxysmal & $245(69)$ & $56(66)$ & $189(70)$ & NS \\
\hline Persistent & $40(11)$ & $11(13)$ & $29(11)$ & NS \\
\hline Permanent & $71(20)$ & $18(21)$ & $53(19)$ & NS \\
\hline \multicolumn{5}{|l|}{ Comorbidities (n (\%)) } \\
\hline $\mathrm{CHF}$ & $34(10)$ & $15(18)$ & $19(7)$ & $p=0.007$ \\
\hline HTN & $164(46)$ & $41(48)$ & $123(45)$ & NS \\
\hline DM & $38(11)$ & $10(12)$ & $28(10)$ & NS \\
\hline Stroke/TIA & $35(10)$ & $10(12)$ & $25(9)$ & NS \\
\hline MIPADAP & $37(10)$ & $14(16)$ & $23(9)$ & $p=0.04$ \\
\hline \multicolumn{5}{|l|}{$\mathrm{CHADS}_{2}$-VASc score (n (\%)) } \\
\hline 0 & $56(16)$ & $10(12)$ & $46(17)$ & NS \\
\hline 1 & $54(15)$ & $18(21)$ & $36(13)$ & NS \\
\hline$\geq 2$ & $242(68)$ & $55(65)$ & $187(69)$ & NS \\
\hline \multicolumn{5}{|l|}{ Medications (n (\%)) } \\
\hline CC-blocker & $72(20)$ & $17(20)$ & $55(20.3)$ & NS \\
\hline B-blocker & $170(48)$ & $40(47)$ & $130(48)$ & NS \\
\hline ARB & $26(7)$ & $8(9)$ & $18(6.6)$ & NS \\
\hline ACE-I & $47(13)$ & $11(13)$ & $36(13)$ & NS \\
\hline$A A D$ & $90(25)$ & $26(31)$ & $64(24)$ & NS \\
\hline ASA & $68(19)$ & $21(25)$ & $47(17)$ & NS \\
\hline DOAC & $166(46)$ & $38(45)$ & $128(48)$ & NS \\
\hline Warfarin & $45(13)$ & $17(20)$ & $28(10$ & $p=0.03$ \\
\hline OAC & $211(59)$ & $55(65)$ & $156(58)$ & NS \\
\hline $\begin{array}{l}\text { Duration of AF before } \\
\text { ED visit in minutes ( } n \text {, } \\
\text { mean } \pm S D \text { ) }\end{array}$ & $717.1 \pm 332$ & $773.7 \pm 301$ & $699.2 \pm 340$ & NS \\
\hline \multicolumn{5}{|l|}{$\begin{array}{l}\text { Thirty-day symptoms ( } \mathrm{n} \\
(\%))^{*}\end{array}$} \\
\hline Yes & $241(68)$ & $62(73)$ & $179(66)$ & NS \\
\hline No & $114(32)$ & $23(27)$ & $91(34)$ & NS \\
\hline \multicolumn{5}{|l|}{ Previous ED Visits (n (\%)) } \\
\hline 0 & $31(9)$ & $10(12)$ & $21(8)$ & NS \\
\hline 1 & $82(23)$ & $17(20)$ & $65(24)$ & NS \\
\hline 2 & $61(17)$ & $11(13)$ & $50(19)$ & NS \\
\hline 3 & $47(13)$ & $15(18)$ & $32(12)$ & NS \\
\hline 4 & $22(6)$ & $4(5)$ & $18(7)$ & NS \\
\hline 5 & $111(31)$ & $28(33)$ & $83(31)$ & NS \\
\hline
\end{tabular}




\begin{tabular}{|c|c|c|c|c|}
\hline & All patients & $\begin{array}{l}\text { Assessed as } \\
\text { appropriate }\end{array}$ & $\begin{array}{l}\text { Assessed as } \\
\text { inappropriate }\end{array}$ & \\
\hline & $n=356$ & $n=85$ & $n=271$ & $P$ value \\
\hline Yes & $94(27)$ & $24(28)$ & $70(26)$ & NS \\
\hline
\end{tabular}

*Symptoms with the 30 days preceding ED attendance related to AF.

$A A D$, antiarrhythmic drug; ACE-I, angiotensin-converting-enzyme inhibitor; AF, atrial fibrillation; ARB, Angiotensin II receptor blocker; ASA, aspirin; CC-blocker, calcium-channel blocker; CHF, congestive heart failure; DM, diabetes mellitus; DOAC, direct oral anticoagulant; ED, emergency department; HTN, hypertension; MIPADAP, myocardial infarction with peripheral arterial disease; OAC, oral anticoagulant; TIA, transient ischaemic attack.

in the appropriate than inappropriate group $(\mathrm{p}<0.0001$ for each symptom).

Conversely, inappropriate ED attendance was significantly correlated to patients receiving medical advice prior to their ED visit $(113 / 271,42 \%$; $\mathrm{p}<0.0001)$.

Notably, significantly more patients who presented for inappropriate reasons were driven by prior medical advice related to the duration of their AF episodes $(18 \%$ vs $1 \%, \mathrm{p}<0.0001)$ or heart rate $(21 \%$ vs $6 \%, \mathrm{p}=0.002)$ as compared with those with appropriate visits. Among patients who were fearful of experiencing further complications, such as stroke, the mean $\mathrm{CHADS}_{2}$-VASc score was 2 and more than $50 \%$ of patients $(37 / 67,55 \%)$ were receiving treatment with an $\mathrm{OAC}$ at baseline presentation.

Education level and annual income category did not impact the appropriateness of the patient's visit to the ED.

A random effects logistic regression model was then conducted to identify which baseline characteristics and presenting patient concerns were significantly associated with inappropriate ED attendance (table 2). This included clinically relevant covariates such as age, sex and anticoagulation status. The model suggested that having an existing history of CHF or attending the ED on the basis of symptoms was significantly associated with a decreased odds of inappropriate ED attendance with an

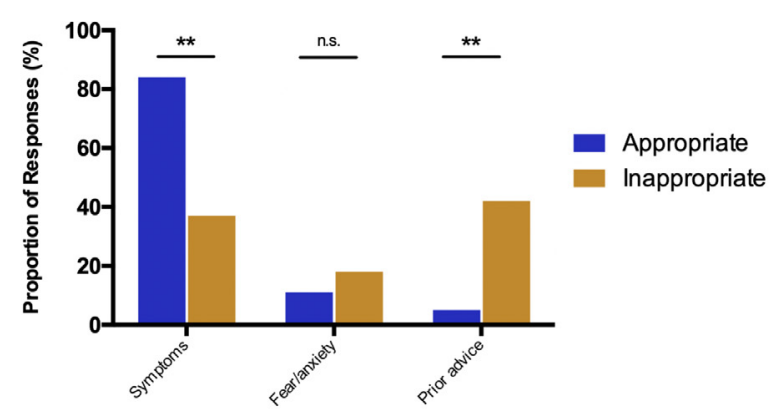

Reason for ED Presentation

Figure 2 Proportion of survey responses of patient-specific reasons for seeking urgent care; ${ }^{* *} p<0.0001$. ED, emergency department.
$\mathrm{OR}=0.3(95 \%$ CI 0.111 to $0.864, \mathrm{p}=0.025)$ and an $\mathrm{OR}=0.1$ (95\% CI 0.00784 to $0.590, \mathrm{p}=0.015$ ), respectively; that is, the attendance was deemed to be more appropriate. No other variables were found to be significantly associated with inappropriate ED attendance in this analysis.

\section{Alternatives to ED management}

Overall, 24\% (85/356) of patients considered their ED attendance absolutely necessary, of which only 22/85 $(26 \%)$ presented for reasons warranting treatment in the ED. Of the $271 / 356(76 \%)$ who felt that their attendance was not necessary 63/271 (23\%) had an attendance that was deemed to be significant. When patients were surveyed regarding potential alternatives to seeking emergency care, the highest proportion of responses among both groups were in favour of an accessible rapid assessment outpatient arrhythmia clinic (186/356, 52\%). The proportion of patient responses for other alternatives including an arrhythmia telephone service, accessibility to an arrhythmia nurse and an AF smart phone application was comparable between the two groups (table 3 ).

\section{Qualitative results}

Impact of AF on general health

The impact of AF on general health status was summarised by most participants mentioning how their daily activities had become limited. Recreational activities such as exercising at the gym, golfing, running, hiking or curling had subsequently decreased or stopped. Some participants also mentioned that their work performance had been adversely affected. In describing living with AF, participants described a markedly decreased quality of life, as much as ' $a 3$ or $2 / 10$ ' and expressed concerns of maintaining relationships with others (figure 3 ).

Participants also mentioned frequently experiencing physical symptoms such as chest pain, chest tightness and shortness of breath during episodes of AF. Experiencing these symptoms was described by many as reasons for attending the ED. Further discussions revealed that panic and anxiety were commonly encountered due to their AF, while several patients expressed their constant fear of having a 'heart attack'. Finally, participants commented 
Table 2 Random intercepts logistic regression analysis for the prediction of inappropriate ED attendance

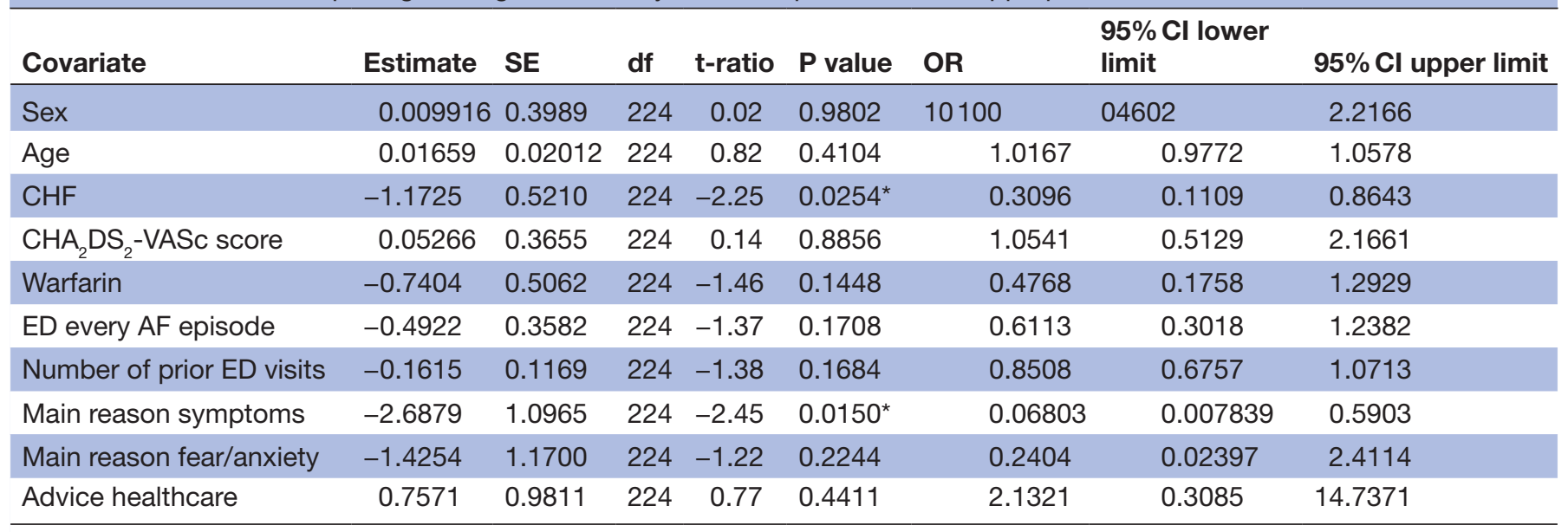

*Denotes significance $p<0.05$.

.AF, atrial fibrillation; $\mathrm{CHF}$, congestive heart failure; ED, emergency department.

on making lifestyle changes to accommodate life with AF. They mentioned a variety of physical activities such as walking short distances and engaging in low-impact aerobics, yoga and staying active. Select patient quotes are shown in figure 3 .

\section{Reasons for ED visitation and outcomes}

Participants described the factors contributing to their decision to attend the ED. As displayed in figure 3, these factors focused largely on their physical symptoms, emotional response, duration of symptoms and prior advice. The majority of participants mentioned experiencing symptoms such as rapid, racing and irregular heart rates, while others recalled feelings of lightheadedness and nausea, or experiencing numbness or swelling in the extremities. For many patients, these physical symptoms came suddenly, with many concerned that they 'would have a heart attack, stroke or complications (clots)'. In addition to AF-related symptoms, many participants reported having overall unpleasant or 'funny feelings' and stated that they intuitively could sense 'something was wrong'.

With respect to emotional responses, many participants recalled feeling scared particularly when these symptoms did not resolve on their own with rest or medications. Fear of dying was consistently mentioned as well as fear of experiencing AF-related complications. Further discussions led to patients explaining that 'The horror of it [experiencing complications] is horrible'. A sizeable portion of participants stated that they had experienced symptoms for more than 1 day prior to coming to the ED, which they attempted to manage on their own but were eventually unsuccessful.

A significant number of participants also mentioned being advised to go to the ER by their physician or another health provider (pharmacist, paramedic and clinic staff) after describing their symptoms and their duration.

\section{DISCUSSION}

To our knowledge, this is the largest prospective, multicentre, mixed methods analysis evaluating patient-specific reasons for inappropriate attendance to the ED based on quantitative and qualitative data.

In order to understand factors associated within appropriate ED attendance, we quantitatively and qualitatively reviewed patient characteristics. The principal findings of this study are that more than $75 \%$ of patients presenting to the $\mathrm{ED}$ with a history of AF did not receive treatment warranting urgent ED care. Factors influencing the inappropriateness of ED visitation included patient decisions based on prior advice prescribed by a healthcare

Table 3 Proportion of patient responses favouring alternative management strategies, stratified by inappropriate versus appropriate visits

\begin{tabular}{llcc}
\hline Alternative strategy & Appropriate $\mathbf{n = 8 5}(\mathbf{n}(\%))$ & Inappropriate $\mathbf{n = 2 7 1}(\mathbf{n}(\%))$ & P value \\
\hline Arrhythmia telephone line & $30(35)$ & $87(32)$ & 0.68 \\
\hline 24/7 arrhythmia nurse & $26(31)$ & $86(32)$ & 0.95 \\
Rapid assessment outpatient clinic & $48(57)$ & $138(51)$ & 0.44 \\
AF smartphone application & $3(4)$ & $16(6)$ & 0.57 \\
No alternative to ED & $22(26)$ & $63(23)$ & 0.73 \\
\hline
\end{tabular}

AF, atrial fibrillation; ED, emergency department. 


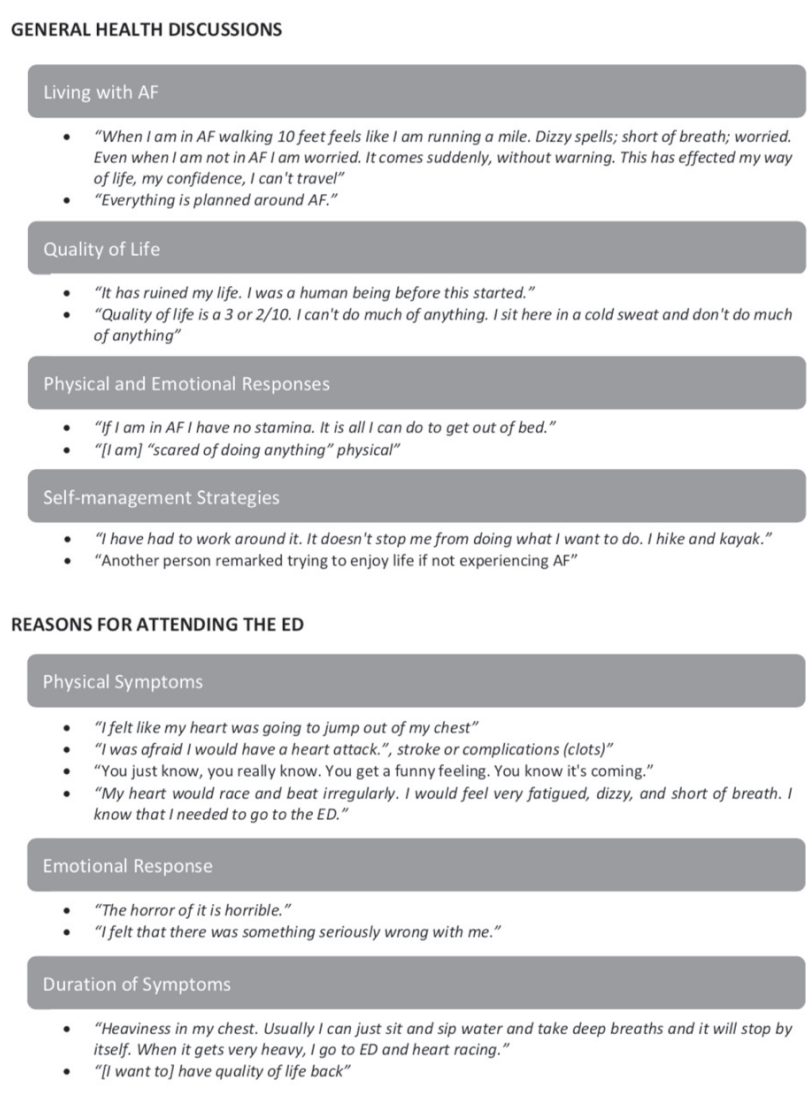

Figure 3 Select patient quotes. AF, atrial fibrillation; ED, emergency department.

professional. In the random effects logistic regression, only two factors appeared to be significantly associated with a decreased odds of inappropriate ED attendance, including an existing history of CHF and patient concerns derived on the basis of AF-related symptoms. Qualitative analysis confirmed these results as physical symptoms, emotional response and prior advice were cited as the predominant reasons for ED visitation. Overall, a marginal proportion $(<25 \%)$ of patients deemed their ED attendance to be absolutely necessary and responded favourably towards alternative treatment strategies, including a rapid assessment outpatient clinic, access to a nurse practitioner and a smartphone application. The highest proportion of responses among both groups was in favour of a specialised rapid assessment outpatient clinic. The results of the current study suggest that many patients may be more effectively managed outside of the ED without compromising the quality or delivery of care and thereby, reducing annual healthcare costs associated with AF-related ED attendances and inappropriate hospitalisations.

AF-related hospitalisations have increased $<2.4$ fold from 1985 to 1999 and by an additional 23\% in the past decade, contributing to the elevated costs incurred from AF management over the years. ${ }^{14}$ Insight into patient perspectives for attending the ED with an existing history of AF therefore have the potential to inform the development of management pathways to promote effective self-management and avoid inappropriate ED attendances altogether. Previous studies have highlighted discrepancies in structured AF management with respect to bridging the gap between ED expertise and integrated AF care in an outpatient setting. Of these, results demonstrated that a significant proportion of ED patients are appropriate for safe and effective outpatient management of $\mathrm{AF}^{15-17}$ These pathways include strategies for early rhythm control, initiation of rate control therapies and anticoagulation for stroke prophylaxis. ${ }^{15} 16$ However, due to barriers in implementation, these strategies have yet to be maintained nor fully accepted. The current results provide evidence for the initiation of rapid assessment outpatient clinics that provide specialised care for $\mathrm{AF}$ symptom management. In a recent meta-analysis, nurse-led AF clinics were found to be more cost-effective, have shorter wait times and reduce subsequent ED visits and hospitalisations. ${ }^{17}$ When compared with standard care, guideline-based medication adherence and patient mortality rates were lower, while quality of life was improved. However, in a study randomising patients with a history of AF to receive nurse-led care or usual care, there was no overall advantage to nurse-led care in a composite of cardiovascular death and cardiovascular hospital admissions. ${ }^{18}$

The current study also reveals a considerable proportion of patients who present to the ED in the absence of symptoms and instead driven predominantly on the basis of generalised fear or prior medical advice. Subsequent content analysis highlighted that patient decisions were driven, largely in part, by their emotional response to their AF episode as well as the lack of self-management strategies that currently exist. Therefore, it is of critical importance to also tailor $\mathrm{AF}$ management strategies towards improving out-of-hospital patient education and providing readily accessible resources. If patients perceive to have rapid access to tools and resources that can guide their acute decision making, they may be less likely to visit the ED altogether.

Of note despite the fact that $68 \%$ of patients had a $\mathrm{CHA}_{2} \mathrm{DS}_{2}$-VASc, score of 2 or more, only $59 \%$ of patients were receiving oral anticoagulation in the form of either a directOAC or warfarin. Additionally, only half of all patients who attended the ED due to a fear a potential stroke were receiving an $\mathrm{OAC}$ despite a mean $\mathrm{CHA}_{2} \mathrm{DS}_{2}$ VASc score of 2. While some of these patients may have not met the criteria for an OAC and some may have had a potential risk of bleeding, there still remains a treatment gap that should be addressed in the initiation of OACs in patients who are considered at a significant risk of stroke, and this may have a positive impact on a reduction in the numbers of patients who attend due to a risk of stroke.

Patients also selected the use of smartphone technology as a viable alternative to usual ED management. Due to the increasing demand for technology in healthcare practices, the ESC, in collaboration with the CATCH ME consortium, has developed smartphone and tablet apps 
for patients and healthcare professionals ${ }^{19}$ as part of the 2016 AF Guidelines. Indeed, the feasibility of mobile technology and remote telephone services has been explored and shown to be associated with increasing efficacy over the years. Previously, the use of telemedicine for rapid out-of-hospital diagnosis and the provision of objective instructions has been assessed as an alternative management pathway for patients with a history of paroxysmal $\mathrm{AF}^{20}{ }^{20}$ Results of this study demonstrated that almost $80 \%$ of paroxysmal AF episodes were managed successfully out of hospital, avoiding the need for unnecessary hospitalisations or clinic visits.

Taken together, future studies should examine novel protocols for outpatient management that involve the integration of technology to enhance the institution of multidisciplinary nurse-led AF assessment clinics and the delivery of patient-centred care.

\section{Limitations}

There are several limitations that should be considered when interpreting the current results and that may be better addressed in future studies. First, the use of a survey-based approach to data collection is susceptible to reporting bias. However, integration of a mixed methods approach permits a more complete and synergistic application of data than quantitative and qualitative data analyses alone. Second, this multicentre study was based on voluntary participation from all centres. Yet, while we sought to achieve geographic variability through an equal distribution of participating centres across provinces, the resulting cohort tended to favour patients attending an academic centre $(81 \%$ academic vs $19 \%$ community). It should also be highlighted that only medium to high expertise centres were approached and eventually enrolled.

The designation of appropriate ED attendance is based on the need for a cardioversion (either electrical or chemical) or an admission to the hospital. While this is based on current guidelines, it could be argued that patients with $\mathrm{AF}$ and an elevated ventricular rate may be considered as an appropriate attendance as they may require treatment with intravenous rate control agents and monitoring. These data are not available for analysis, and this should be considered a limitation of the study.

Only participants who returned a completed consent form following their discharge from the ED were approached to take part in this study. Additionally, 19\% of patients who were approached for this study declined to take part, which may have altered the distribution of responses. While the recruitment of patients was difficult at times based on institution-specific approaches to accessing and documenting ED data, each centre had high response rates that avoided the need for multiple sampling over time. The AF-ED multicentre trial still remains the largest Canadian multicentre mixed methods assessment of patient-specific motivators for ED attendance in those with a history of AF. Therefore, while several noteworthy conclusions can be drawn from these results, future studies should focus on devising novel outof-hospital protocol for integrated AF management and self-management techniques focused on both symptom reduction and improving emotional well-being.

\section{CONCLUSIONS}

Given the epidemic proportions of AF incidence and prevalence predicted for the coming decades, outpatient rapid assessment pathways and improved patient education may provide optimal treatment alternatives for $\mathrm{AF}$, thereby reducing the need for consuming valuable and limited healthcare resources in the ED.

\section{Author affiliations}

${ }^{1}$ Department of Cardiology, Sunnybrook Health Sciences Centre, Toronto, Ontario, Canada

${ }^{2}$ Department of Medicine, University of Toronto, Toronto, Ontario, Canada ${ }^{3}$ Department of Cardiology, Queen's University, Kingston, Ontario, Canada ${ }^{4}$ Department of Cardiology, Interior Health Authority, Kelowna, Province of British Columbia, Canada

${ }^{5}$ Department of Emergency Medicine, The University of British Columbia, Vancouver, British Columbia, Canada

${ }^{6}$ Department of Emergency Medicine, Nova Scotia Health Authority, Halifax, Province of Nova Scotia, Canada

${ }^{7}$ Department of Cardiology, University of Alberta, Edmonton, Western Canada, Canada

${ }^{8}$ Wilkes Honors College, Florida Atlantic University, Boca Raton, Florida, USA

${ }^{9}$ Division of Cardiology, London Health Sciences Centre, London, Ontario, Canada

Twitter Kathryn Lauren Hong @kathrynhong

Acknowledgements We would like to acknowledge Ms Lisa Carver for her help in this study.

Contributors KLH, CB, BZ, MB, SH, FH, CMH, KM, RKS, SYT, AK, TL, ZM, PD, PA, $M E$ and PL-S were involved in the planning, conduct and reporting of the work in this article. BMG was the principle investigator and was involved in the planning, conduct and reporting of the work in this article. We would like to acknowledge Ms Lisa Carver for her help in this study. All authors meet the ICNJE criteria.

Funding This study was supported by the Cardiac Arrhythmia Network of Canada. The study sponsor had no influence on the interpretation of these results.

Competing interests None declared.

Patient and public involvement Patients and/or the public were involved in the design, or conduct, or reporting, or dissemination plans of this research. Refer to the Methods section for further details.

Patient consent for publication Not required.

Ethics approval This study was reviewed for ethical compliance by all institutional research ethics boards and conducted in keeping with international quality standards that have been adopted by Health Canada.

Provenance and peer review Not commissioned; externally peer reviewed.

Data availability statement All data relevant to the study are included in the article or uploaded as supplementary information. Statistical code and full dataset available from the corresponding author at benedict.glover@sunnybrook.ca.

Open access This is an open access article distributed in accordance with the Creative Commons Attribution Non Commercial (CC BY-NC 4.0) license, which permits others to distribute, remix, adapt, build upon this work non-commercially, and license their derivative works on different terms, provided the original work is properly cited, appropriate credit is given, any changes made indicated, and the use is non-commercial. See: http://creativecommons.org/licenses/by-nc/4.0/.

\section{ORCID iDs}

Kathryn Lauren Hong http://orcid.org/0000-0001-6229-1323

Sohaib Haseeb http://orcid.org/0000-0003-2799-5135

Simon Yu Tian http://orcid.org/0000-0002-0706-0757 


\section{REFERENCES}

1 Wilke T, Groth A, Mueller S, et al. Incidence and prevalence of atrial fibrillation: an analysis based on 8.3 million patients. Europace 2013;15:486-93.

2 Atzema CL, Austin PC, Miller E, et al. A population-based description of atrial fibrillation in the emergency department, 2002 to 2010. Ann Emerg Med 2013;62:570-7.

3 Chugh SS, Blackshear JL, Shen WK, et al. Epidemiology and natural history of atrial fibrillation: clinical implications. J Am Coll Cardiol 2001;37:371-8.

4 Go AS, Hylek EM, Phillips KA, et al. Prevalence of diagnosed atrial fibrillation in adults: national implications for rhythm management and stroke prevention: the anticoagulation and risk factors in atrial fibrillation (atria) study. JAMA 2001;285:2370-5.

5 Naccarelli GV, Varker H, Lin J, et al. Increasing prevalence of atrial fibrillation and flutter in the United States. Am J Cardiol 2009;104:1534-9.

6 McDonald AJ, Pelletier AJ, Ellinor PT, et al. Increasing us emergency department visit rates and subsequent hospital admissions for atrial fibrillation from 1993 to 2004. Ann Emerg Med 2008;51:58-65.

7 Barbic D, DeWitt C, Harris D, et al. Implementation of an emergency department atrial fibrillation and flutter pathway improves rates of appropriate anticoagulation, reduces length of stay and thirty-day revisit rates for congestive heart failure. CJEM 2018;20:392-400.

8 Humphries KH, Jackevicius C, Gong Y, et al. Population rates of hospitalization for atrial fibrillation/flutter in Canada. Can J Cardiol 2004;20:869-76.

9 Singer DE, Chang Y, Fang MC, et al. The net clinical benefit of warfarin anticoagulation in atrial fibrillation. Ann Intern Med 2009;151:297-305.

10 O'Reilly DJ, Hopkins RB, Healey JS, et al. The burden of atrial fibrillation on the hospital sector in Canada. Can $J$ Cardiol 2013;29:229-35.
11 Stiell IG, Macle L. Canadian cardiovascular Society atrial fibrillation guidelines 2010: management of recent-onset atrial fibrillation and flutter in the emergency department. Can J Cardiol 2011;27:38-46.

12 Macle L, Cairns JA, Andrade JG, et al. The 2014 atrial fibrillation guidelines companion: a practical approach to the use of the Canadian cardiovascular Society guidelines. Can J Cardiol 2015;31:1207-18.

13 Kirchhof P, Benussi S, Kotecha D, et al. Esc guidelines for the management of atrial fibrillation developed in collaboration with EACTS: the task force for the management of atrial fibrillation of the European Society of cardiology (ESC) developed with the special contribution of the European heart rhythm association (EHRA) of the $\mathrm{Sc}$ endorsed by the European stroke organisation (ESO). Europace 2016;2016:1609-78.

14 Ptaszek LM, White B, Lubitz SA, et al. Effect of a multidisciplinary approach for the management of patients with atrial fibrillation in the emergency department on hospital admission rate and length of stay. Am J Cardiol 2016;118:64-71.

15 White JL, Heller MB, Kahoud RJ, et al. Performance of an expedited rhythm control method for recent onset atrial fibrillation in a community hospital. Am J Emerg Med 2015;33:957-62.

16 Carter L, Gardner M, Magee K, et al. An integrated management approach to atrial fibrillation. J Am Heart Assoc 2016;5:e002950.

17 Rush KL, Burton L, Schaab K, et al. The impact of nurse-led atrial fibrillation clinics on patient and healthcare outcomes: a systematic mixed studies review. Eur J Cardiovasc Nurs 2019;18:526-33.

18 Witvliet E, Tieleman RG, van Gelder IC, et al. usual-care for atrial fibrillation. Eur Heart J 2019.

19 Kotecha D, Chua W, Fabritz L, et al. European Scoiety of cardiology (ESC) smartphone and tablet applications for patients with atrial fibrillation and their healthcare providers. Europace 2017.

20 Shacham J, Birati EY, Malov N, et al. Telemedicine for diagnosing and managing paroxysmal atrial fibrillation in outpatients. The phone in the pocket. Int J Cardiol 2012;157:91-5. 